\title{
Vereinfachte Regel erlaubt Ausschluss tiefer Venenthrombosen
}

Xu K et al. A simplified decision rule to rule out deep vein thrombosis using clinical assessment and D-dimer. J Thromb Haemost 2021; 19: 1752-1758. doi: $10.1111 /$ jth. 15337

Patienten mit Symptomen einer Thrombose der tiefen Beinvenen wie Schwellungen und Rötungen des betroffenen Unterschenkels stellen in der Allgemeinpraxis oder der Notaufnahme keine Seltenheit dar. Normalerweise besteht das Standardvorgehen zunächst in der klinischen Beurteilung, wie wahrscheinlich bei dem jeweiligen individuellen Patienten eine tiefe Venenthrombose (TVT) ist. Erst danach folgt ggf. die Bestimmung der D-Dimer-Konzentration.

Eine Möglichkeit, diese Wahrscheinlichkeit (klinische Vortestwahrscheinlichkeit) abzuschätzen, bietet der sog. Wells DVT Clinical Sore mit insgesamt 9 Items. Allerdings ist die Berechnung dieses Werts oftmals kompliziert, da mehrere Subkomponenten einbezogen werden müssen daher wird er in der Alltagspraxis oftmals gar nicht eingesetzt oder falsch berechnet. Eine schwedisch-kanadische Arbeitsgruppe hat nun versucht, eine einfachere Regel zu entwickeln.

Keying Xu und Kollegen haben insgesamt 4 prospektive Studien mit 3368 Patienten in ihre Analyse einbezogen. Bei allen Teilnehmern bestand der Verdacht auf eine TVT und es war eine quantitative Messung der D-Dimer-Konzentration erfolgt. Nach dem Original-Wells-Score hatte bei 34\% eine geringe, bei $42 \%$ eine moderate und bei $24 \%$ eine hohe klinische Vortestwahrscheinlichkeit für eine TVT bestanden. Insgesamt war bei $17 \%$ eine TVT bestätigt worden, entweder bei der Ausgangsuntersuchung oder bei späteren Kontrollen.

Die Wissenschaftler errechneten in mehreren Schritten aus den Original-Patientendaten in einem Regressionsmodell diejenigen Wells-Kriterien, die am besten zum Ausschluss einer TVT geeignet waren; „sicher ausgeschlossen“ war hier definiert als oberer Grenzwert des 95\%-Konfidenzintervalls $(95 \%-\mathrm{KI})<2 \%$.

Zwei Kriterien erwiesen sich als am aussagekräftigsten:

- Schwellung des Unterschenkels $\geq 3 \mathrm{~cm}$ und

- die Einschätzung einer TVT als wahrscheinlichste Diagnose (eine Umkehrung des „eine andere Diagnose ist wahrscheinlicher als eine TVT“).

Darüber hinaus legten sie $3 \mathrm{D}$-DimerGruppen fest:

- <500 ng/ml,

- 500-999 ng/ml und

- $\geq 1000 \mathrm{ng} / \mathrm{ml}$.

Anhand der oben genannten Kriterien definierten die Forscher dann 2 Gruppen:

- geringe klinische Vortestwahrscheinlichkeit: keines der Kriterien liegt vor sowie

- hohe klinische Vortestwahrscheinlichkeit: $\geq 1$ Kriterium liegt vor.

Dann wendeten sie diese Kombination von Klinik und Labor auf die Originaldaten an:

Bei „geringe klinische Vortestwahrscheinlichkeit" ( $n=920)$ fand sich eine TVT bei insgesamt 63 Patienten (6,8\%); aufgeschlüsselt nach D-Dimer-Subgruppen waren es

- $0,1 \%$ bei einem D-Dimer-Wert $<500 \mathrm{ng} / \mathrm{ml}$ und

- $0,3 \%$ bei einem D-Dimer-Wert von 500-999 ng/ml.

In beiden Fällen wurde der festgelegte obere Grenzwert des 95\%-KI nicht überschritten, sodass eine TVT sicher ausgeschlossen werde konnte.
Bei „hohe klinische Vortestwahrscheinlichkeit" ( $n=2448$ ) bestand eine TVT bei insgesamt 520 Patienten (21,2\%); aufgeschlüsselt nach D-Dimer-Subgruppen waren es

- $0,3 \%$ bei einem D-Dimer-Wert $<500 \mathrm{ng} / \mathrm{ml}$ und

- 5,8\% bei einem D-Dimer-Wert von 500-999 ng/ml.

Für Patienten mit einer D-Dimer-Konzentration $<500 \mathrm{ng} / \mathrm{ml}$ war eine TVT damit ausgeschlossen, bei Werten zwischen 500 und $999 \mathrm{ng} / \mathrm{ml}$ allerdings lag das $95 \%-\mathrm{KI}$ bei 1,9-9,7), sodass in diesem Fall eine BVT nicht sicher ausschließbar war.

Aus den Daten errechneten sich für die Kombination der 3 Parameter schließlich

- ein negativer Vorhersagewert von $99,3 \%$,

- eine Spezifität von 49,4\% und

- eine Utility von $40,6 \%$.

FAZIT

Insgesamt konnte ihre Regel mit nur 3 Parametern (Unterschenkelschwellung, TVT wahrscheinlichste Diagnose, D-Dimer-Konzentration) bei den verwendeten Patientendaten in $43 \%$ der Fälle eine TVT sicher ausschließen, so die Autoren. Damit unterscheidet sich die Genauigkeit zwar nicht wesentlich von der des Wells-Scores, die von den Autoren „DAY-Regel“ genannte ist aber wesentlich weniger umständlich. Allerdings müsste sie noch extern prospektiv an größeren Patientengruppen validiert werden.

Dr. Elke Ruchalla, Bad Dürrheim 